\title{
Mode Selection Schemes for Unicasting Device-to-Device Communications Supported by Network Coding
}

\author{
Gábor Fodor \\ Ericsson Research and Royal Institute of Technology \\ Gabor.Fodor@ericsson.com; gaborf@kth.se
}

\begin{abstract}
Device-to-device (D2D) communication in a cellular spectrum increases the spectral and energy efficiency of local communication sessions, while also taking advantage of accessing licensed spectrum and higher transmit power levels than when using unlicensed bands. In order to realize the potential benefits of D2D communications, appropriate mode selection algorithms that select between the cellular and D2D communication modes must be designed. On the other hand, physical layer network coding (NWC) at a cellular base station - which can be used without D2D capability - can also improve the spectral efficiency of a cellular network that carries local traffic. In this paper, we ask whether cellular networks should support D2D communications, physical layer NWC, or both. To this end, we study the performance of mode selection algorithms that can be used in cellular networks that employ physical layer NWC and support D2D communications. We find that the joint application of D2D communication and NWC scheme yields additional gains compared to a network that implements only one of these schemes, provided that the network implements proper mode selection and resource allocation algorithms. We propose two mode selection schemes that aim to achieve high signal-tointerference-plus-noise ratio and spectral efficiency, respectively, and take into account the NWC and D2D capabilities of the network. ${ }^{1}$
\end{abstract}

\section{INTRODUCTION}

Device-to-device (D2D) communication in a cellular spectrum assisted by a cellular network enables direct communication between user equipments (UE) that are in the proximity of one another $[2,3]$. The objective of supporting D2D communication in a licensed spectrum assisted by cellular networks is to exploit the so-called reuse and proximity gains [3] of UEs when engaged in proximal communication sessions such as social aware communications [4], vehicle or machine type communication, or proximity-based services [5]. However, the current release of the 3GPP standards suite only supports broadcasting at the physical and medium access control layers, which does not allow a D2D transmitter to adjust its transmit parameters to an intended peer receiver.

When proximate communication opportunities exist, unicasting D2D communication has advantages over the traditional cellular communication mode that routes local traffic through the cellular base station and uses both uplink and downlink resources. Indeed, D2D communication increases the spectral

\footnotetext{
${ }^{1}$ An early version of this manuscript was presented at the European Wireless conference in May 2014 (author?) [1].
}

efficiency not only due to the proximity gain in terms of improved link budget, but also due to the so-called spectrum reuse gain and hop gain [3], [6-9]. A necessary technology component of D2D is mode selection (MS), which selects the cellular or direct communication mode for a D2D pair based on factors such as the large or small-scale fading between the communicating devices, as well as between the devices and the cellular base station, traffic load, and interference level [9]. Recognizing the potential of unicasting D2D, previous works have proposed efficient mode selection, resource allocation, and power control algorithms that help realize the proximity, reuse, and hop gains of local communications, while protecting both the cellular and D2D layers from interference that arises due to the tight spectrum reuse [10].

Recognizing the high potential of D2D communications, the 3rd Generation Partnership Project (3GPP) has recently included control plane and measurement support for physical layer broadcasting based D2D communications [11]. The 3GPP is currently studying the necessary physical layer enhancements for introducing physical layer unicast support for D2D communications in the future releases of Long Term Evolution (LTE) and New Radio (NR) networks [12]. Indeed, physical layer unicast - , that is, the D2D transmitter being aware of the intended receiver, as opposed to the currently supported broadcast communication -, is needed to fully realize the gains expected from introducing D2D communications in a cellular spectrum.

Studies have shown that when local (proximal) communication opportunities exist in a cellular network, physical layer network coding (NWC) improves the spectrum efficiency by enabling resource reuse by multiple transmissions and cancelling harmful interference using advanced signal processing techniques $[13,14]$. Despite the large differences between D2D communications assisted by a cellular network and employing various forms of NWC, their ultimate objective of improving the spectral efficiency and increasing the network capacity by enabling tighter reuse of resources is comparable.

Since both technologies have similar objectives, it is natural to ask whether the joint application of D2D and NWC would result in further spectral or energy efficiency gains in a network that supports only one of these techniques. This question initially raised in [1] and [15] - is motivated by the realization that introducing D2D and NWC could be costly in terms of UE 
capabilities, measurement reports, and control plane support.

Therefore, we aim in this article, to answer the following questions:

- Does physical layer NWC provide gains in cellular networks that support unicasting D2D communication, that is, when a D2D transmitter is aware of its intended receiver?

- Does unicasting D2D provide gains in a cellular network that employs physical layer NWC?

Thus, the contribution of our work is that it identifies the possible joint D2D and NWC schemes, and - using system simulations - provides insights into the potential benefits of using them separately or jointly. We also believe that the idea of using a NWC-aware mode selection scheme in D2D-capable cellular network is an important contribution. To this end, we structure the remainder of the paper as follows. Section III discusses the possible transmission modes in an integrated D2D-cellular network that can employ different forms of NWC. Section IV develops a system model and discusses the key performance aspects. In Section V we propose mode selection and resource allocation schemes applicable in the integrated D2D-NWC environment. Section VI discusses numerical results, and Section VII summarizes our findings.

\section{RELATED WORKS AND CONTRIBUTION}

While many papers are closely related to D2D communications in cellular networks, mode selection and NWC facilitated by $\mathrm{D} 2 \mathrm{D}$ communications, our literature survey shows that there have not been any previous studies of mode selection algorithms taking into account both D2D communications and NWC. In particular, the question formulated at the end of Section I (do combined NWC and D2D schemes provide gains over systems employing only one of these techniques) remain unanswered in the literature. We seek an answer to this question, since modern cellular networks are increasingly required to support local (proximity) communications either using D2D communications or NWC or combined schemes.

\section{A. Papers Related to Physical Layer Network Coding}

The seminal paper by [13] gives an in-depth performance analysis and comparison of the two-, three- and four- time slot physical layer network coding (PNC) schemes in terms of the received SNR, outage probabilities and bit error rates. That paper assumes perfect CSI knowledge and does not consider any interference at the receivers. Channel estimation errors and the impact of power allocation on the performance of traditional as well as PNC are examined in [16]. That paper proposes simple power allocation techniques that are well suited to both perfect- and imperfect CSI conditions and high and low SNRs (in interference-free scenarios) and rely on channel statistics. The impact of co-channel interference on the performance of the two-way relay system including the two-, and three-time slot schemes is studied in [14]. More recently, [17] investigated the impact of outdated channel estimates on two-way PNCbased relaying without interference. Although the results and methodologies developed in these papers provide valuable insights, they do not easily generalize to scenarios in which multi-cell interference is present and both cellular and D2D modes are available for proximity communications.

\section{B. Papers Related to Mode Selection for D2D Communica- tions}

Both academia and industry have studied transmission mode selection for D2D communications underlaying a cellular network, see, for example, [18-23]. In those publications, the transmission mode refers to the D2D mode where two D2D UEs communicate directly over the air or to the cellular mode where two D2D UEs communicate via the BS, as in traditional cellular networks. However, as mentioned in [24], the definition of the transmission mode can also be more complex and reflect more design alternatives in D2D communications, including how spectrum sharing is managed between the cellular and D2D users (orthogonal or overlapping resources) and the time scale over which the resources are assigned to D2D users. However, none of these studies include the PNC schemes that are included in the definition of the transmission mode in the present paper. Therefore, these mode selection schemes are not applicable in D2D networks that combine D2D capability with PNC.

\section{Papers Related to D2D Communications Using Network Coding}

Employing network coding in cellular network assisted D2D communication is straightforward and appealing, since the cellular base station can act as a relay between the two communicating UEs. Recognizing the applicability of network coding in D2D communications, several papers have investigated the performance benefits of NWC specifically in D2D communication scenarios [25-29]. The results reported in [25] indicate that direct D2D communication with NWC can use more resources than D2D communication without NWC (depending on the specific NWC scheme), and its application can be beneficial in terms of link quality and communication range. Physical layer NWC-aided two-way D2D communication is considered in [27]. In that paper the D2D system is modeled as a coalition game, and a distributed resource allocation algorithm based on coalition formation is proposed. Reference [29] advocates the use of NWC as an enabling technology for enhanced security and communication efficiency. However, mode selection schemes are beyond the scope of these papers.

\section{Contributions of the Present Paper}

In this paper we develop a model for D2D networks that support two-slot and three-slot (with/without maximum ratio combining) NWC, in addition to traditional cellular communication and D2D communication without NWC. By implementing this model in a realistic multicell system simulator, 


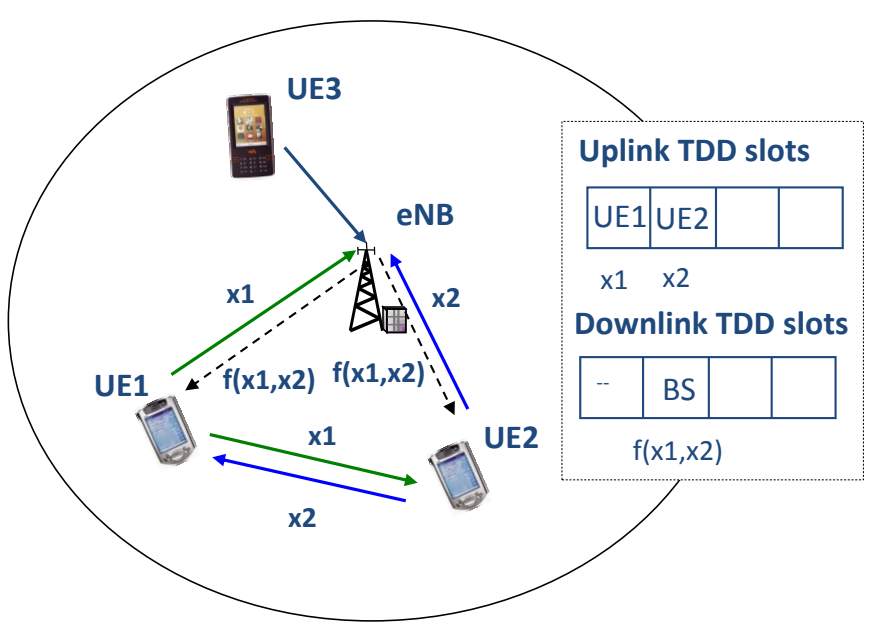

Fig. 1. Unicasting D2D and NWC technologies employed jointly in a cellular network. In uplink TS-1, UE1 transmits $x_{1}$. In uplink TS-2, UE2 transmits $x_{2}$ and finally in downlink TS-3, the eNB transmits the network coded data $f\left(x_{1}, x_{2}\right)$.

and analyzing numerical results, we address two research questions:

- What are the performance gains in terms of the achieved SINR of combined D2D and NWC schemes as compared to a cellular system that supports only one of these schemes?

- What transmission mode selection algorithms should be used in systems that support both D2D and NWC ?

This study contributes to the existing literature through the two proposed mode selection algorithms and engineering insights offered by the associated numerical results. We believe that these results are useful for the research and especially for the standardization community in developing D2D technology enablers that are useful in practice.

\section{EMPLOYing D2D AND NWC to Support LOCAL TRAFFIC}

To understand the similarities and differences between D2D and NWC based operation, consider Figure 1. In the scenario of Figure 1, the D2D-capable UE1 and UE2 are served by the same base station (eNB) while exchanging data with one another. Unicasting D2D-capability enables direct communication without involving the serving eNB, in which case a bidirectional exchange of signals $x_{1}$ and $x_{2}$ requires two orthogonal resources. ${ }^{2}$ For example, when using time division duplexing (TDD) on the D2D link, $x_{1}$ and $x_{2}$ are exchanged in subsequent time slots (TS-1 and TS-2).

Alternatively, in a cellular network in which the eNB uses physical layer NWC, two time slots are sufficient for the exchange of $x_{1}$ and $x_{2}$. That is, using physical layer or 2 time slot (2-TS) NWC, UE1 and UE2 transmit on the same resource (TS-1), while the eNB uses TS-2 to transmit the network coded data $f\left(x_{1}, x_{2}\right)$ to UE1 and UE2 at the same time $[13,14]$. UE1

\footnotetext{
${ }^{2}$ In this paper we do not consider the application of full-duplex communication.
}

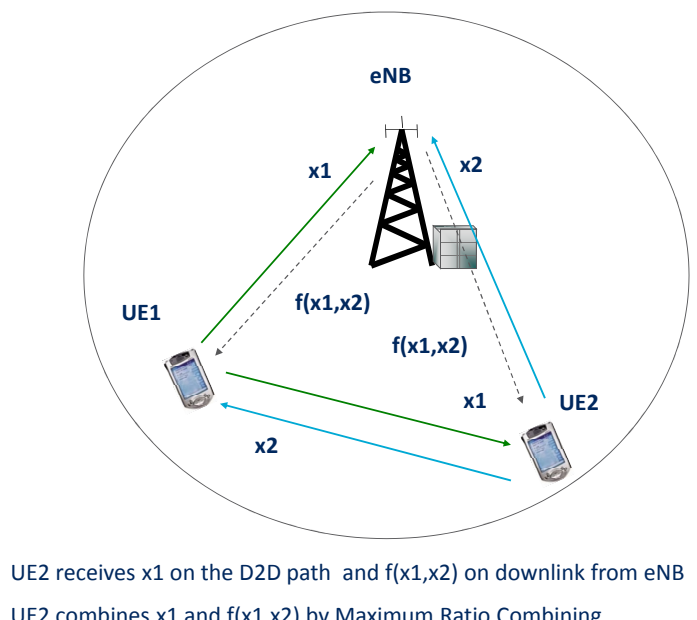

Fig. 2. Maximum ratio combining at UE2: in the uplink time slot (TS-1), UE1 transmits $x_{1}$, which is captured by the eNB and UE2. In the subsequent uplink time slot (TS-2), UE2 transmits $x_{2}$. Finally in downlink time slot (TS-3), the eNB transmits the network coded data $f\left(x_{1}, x_{2}\right)$.

and UE2 use cellular links to receive $f\left(x_{1}, x_{2}\right)$ and decode $x_{2}$ and $x_{1}$, respectively.

As an alternative to the 2-TS NWC scheme, the 3-TS NWC scheme uses orthogonal resources (time slots) to transmit $x_{1}$ and $x_{2}$ to the eNB, while the eNB uses a single time slot (TS-3) to transmit the network-coded data of $f\left(x_{1}, x_{2}\right)$. When UE1 and UE2 are in the proximity of one another, and the eNB supports the 3-TS NWC scheme, it is possible to employ D2D and NWC jointly 1. In this joint NWC-D2D mode, UE2 uses D2D communications to receive the direct transmission from UE1 (in the uplink time slot TS-1) and the network coded transmission from the eNB (in the downlink time slot TS-2). To properly decode the data transmitted by the peer UE, UE2 can then employ signal processing (for example, maximum ratio combining with maximum likelihood detection, as illustrated by Figures 2 and 3) to separate the own transmitted packet from the packet transmitted by UE1.

To understand the combined operation of physical layer network coding at the eNB and maximum ratio combining at the UE2, consider Figure 2 and Figure 3. In order to be able to combine the received signal on the direct D2D link $\left(y_{1}\right)$ and the network coded data on the downlink $\left(y_{2}\right)$, UE2 continuously maintains the corresponding channel estimates $h_{12}$ (D2D link) and $h_{2}$ (downlink). The received D2D and downlink signals at UE2 can then be written as:

$$
\begin{aligned}
& y_{1}=h_{12} x_{1}+n \\
& y_{2}=h_{2} f\left(x_{1}, x_{2}\right)+n,
\end{aligned}
$$

where $n$ denotes the thermal noise at UE2. The input variables to the maximum likelihood decision unit at UE2 are given as:

$$
\begin{aligned}
\hat{x}_{1} \triangleq h_{12}^{\star} y_{1} & =h_{12}^{\star} h_{12} x_{1}+h_{12}^{\star} n \\
\hat{x}_{2} \triangleq h_{2}^{\star} y_{2} & =h_{2}^{\star} h_{2} f\left(x_{1}, x_{2}\right)+h_{2}^{\star} n
\end{aligned}
$$




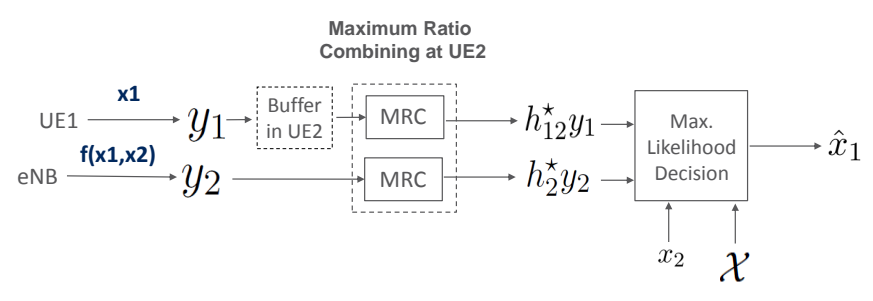

$$
\hat{x}_{1}=\underset{x_{i} \in \mathcal{X}}{\arg \min }\left(\left|y_{1}-h_{12} x_{i}\right|^{2}+\left|y_{2}-h_{2} f\left(x_{i}, x_{2}\right)\right|^{2}\right)
$$

Fig. 3. Details of the maximum ratio combining with maximum likelihood decision at UE2: UE2 uses the received signal through the direct D2D path $\left(y_{1}\right)$, and the received network coded data $\left(y_{2}\right)$ to estimate the transmitted symbol $x_{1} \in \mathcal{X}$, where $\mathcal{X}$ is is the symbol alphabet.

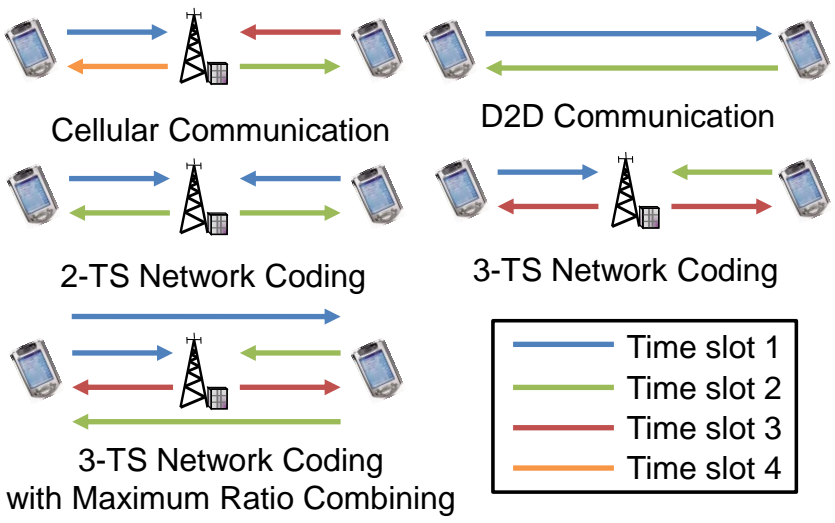

Fig. 4. An overview of the available transmission modes for local communications. The fifth transmission mode (3-TS NWC with maximum ratio combining) integrates D2D and NWC in a joint scheme, as illustrated by Figure 2 and Figure 3.

UE2 uses the MLD rule to estimate the transmitted symbol by $\hat{x}_{i} \in \mathcal{X}$, where $\mathcal{X}$ is the symbol alphabet, as:

$$
\begin{aligned}
\hat{x}_{i} & =\arg \min _{x_{i} \in \mathcal{X}}\left(\left|y_{1}-h_{12} x_{i}\right|^{2}+\left|y_{2}-h_{2} f\left(x_{i}, x_{\text {own }}\right)\right|^{2}\right)= \\
& =\arg \min _{x_{i} \in \mathcal{X}}\left(\left|y_{1}\right|^{2}+\left|y_{2}\right|^{2}+\left|h_{12}\right|^{2}\left|x_{i}\right|^{2}+\left|h_{2}\right|^{2}\left|f\left(x_{i}, x_{\text {own }}\right)\right|^{2}\right. \\
& \left.-x_{i}^{\star} \hat{x}_{1}-x_{i} \hat{x}_{1}^{\star}-\hat{x}_{2} f^{\star}\left(x_{i}, x_{\text {own }}\right)-\hat{x}_{2}^{\star} f\left(x_{i}, x_{\text {own }}\right)\right)
\end{aligned}
$$

Note that using the locally available channel estimates and (3)-(4), all six terms that are needed in the MLD above are available at UE2, since UE2 substitutes its own transmitted symbol $x_{2}$ into $x_{\text {own }}$ and the network coding function $f(\cdot, \cdot)$ is known by UE2.

Figure 4 illustrates the possible transmission modes that are available for local (proximity) communications by the traditional cellular, unicasting D2D, and physical layer NWC schemes. Notice that the traditional cellular transmissions
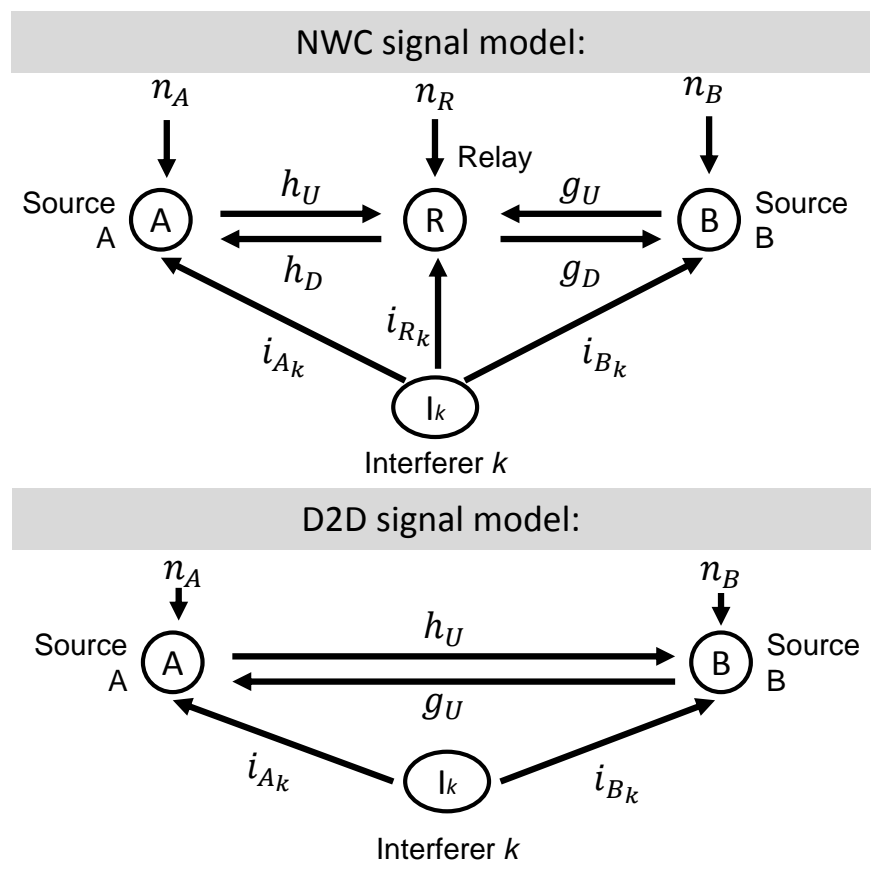

Fig. 5. Signal models for NWC and D2D transmissions. Source A transmits signal $x_{A}$ with power $P_{A}$ while source $\mathrm{B}$ transmits signal $x_{B}$ with power $P_{B}$. NWC transmission involves a relay node (in this case is the base station) while D2D transmission does not. The relay transmits with power $P_{R}$.

(without D2D and NWC capabilities) of $x_{1}$ and $x_{2}$ require four time slots (4-TS), since transmitting each requires an uplink and a downlink time slot [13].

\section{Performance Aspects}

Investigating the performance of the available local communication schemes (Figure 4) analytically is difficult due to the random positions of the UEs and the resulting interference situation at each receiver. Therefore, in this paper we resort to a realistic system simulator to analyze system performance. The performance indicators of interest include the end-toend signal-to-interference-and-noise ratio (SINR), the total transmit power needed for the bidirectional transmissions $\left(P_{A}+P_{B}+P_{R}\right)$, and the resulting spectral efficiency as a function of the achieved SINR and the number of required time slot).

\section{A. Signal Models}

The signal models for the NWC-based and D2D-based transmissions are shown by Figure 5, where the aggregate interference affecting both the end devices (UE1 and UE2) and the relaying equipment (eNB) are also illustrated. In this figure, $h$ and $g$ denote the complex channel coefficients, while $n$ denotes the additive Gaussian noise.

1) Network Coding: As indicated in the upper part of 5, when NWC is employed, the communication is assisted by a relay node that forwards the information from source A to source B and vice versa. In a local communication session, it 
is assumed that sources A and B are UEs while the relay node is the base station (eNB).

Each node receives additional Gaussian noise $n_{A}, n_{B}, n_{R} \sim$ $\mathcal{C N}\left(0, \sigma^{2}\right)$, and experiences interference from other transmitters utilizing the same resource.

Notations $h_{U}, g_{U}, h_{D}, g_{D}, i_{A_{k}}, i_{R_{k}}$ and $i_{B_{k}}$ represent channel gains. In our model, the uplink and downlink channels can have different channel gains.

2) D2D Communication: The signal model for a unicasting D2D communication session is illustrated by the lower part of Figure 5. In this case, the UEs communicate with one other through the direct D2D links, and there is no relay node (eNB) assisting the information exchange.

\section{B. SINR Analysis}

The SINR analysis for the physical NWC schemes is based on [13]. The notations $x_{A}, x_{B}$, and $x_{I_{k}}$ represent data symbols transmitted by source $\mathrm{A}$, source $\mathrm{B}$, and interferer $k$ respectively. The transmit power levels of source A, source B, the relay, and interferer $k$ are denoted as $P_{A}, P_{B}, P_{R}$, and $P_{I_{k}}$ respectively. Furthermore, we define $P_{I_{k}}^{(t)}, i_{R_{k}}^{(t)}, i_{A_{k}}^{(t)}, i_{B_{k}}^{(t)}$, and $x_{i_{k}}^{(t)}$ as the transmit power, channel gains, and data symbols associated with interferer $k$ in time slot $t$.

It is important to realize that the SINR used in this context is different from the traditional single-link SINR. Specifically, we use end-to-end SINR which accounts for both the source-torelay and the relay-to-destination SINRs to better characterize the different transmission schemes. The expressions of all transmission schemes listed in Figure 4 are given below, while the derivations are given in the Appendix.

1) Two-Time Slot (2-TS) Network Coding: For the 2-TS NWC scheme, the end-to-end SINR values at sources A and $\mathrm{B}$ are calculated as follows.

$$
\begin{gathered}
\gamma_{A}=\frac{G^{2} P_{R}\left|h_{D}\right|^{2} P_{B}\left|g_{U}\right|^{2}}{G^{2} P_{R}\left|h_{D}\right|^{2} \psi_{2}+\sum_{k} P_{I_{k}}^{(2)}\left|i_{A_{k}}^{(2)}\right|^{2}+\sigma^{2}} \\
\gamma_{B}=\frac{G^{2} P_{R}\left|g_{D}\right|^{2} P_{A}\left|h_{U}\right|^{2}}{G^{2} P_{R}\left|g_{D}\right|^{2} \psi_{2}+\sum_{k} P_{I_{k}}^{(2)}\left|i_{B_{k}}^{(2)}\right|^{2}+\sigma^{2}} \\
\psi_{2}=\left(\sum_{k} P_{I_{k}}^{(1)}\left|i_{R_{k}}^{(1)}\right|^{2}+\sigma^{2}\right)
\end{gathered}
$$

$G$ is the gain by which the received signals from the sources are amplified at the relay. For 2-TS network coding, $G$ is given by

$$
G=\sqrt{\frac{1}{P_{A}\left|h_{U}\right|^{2}+P_{B}\left|g_{U}\right|^{2}+\sigma^{2}}}
$$

2) Three-Time Slot (3-TS) Network Coding: As a unique property of the 3-TS network coding, the transmit power at the relay is characterized by power allocation factors $\alpha_{A}$ and $\alpha_{B}$, which must be known at both sources [13]. These power allocation factors are determined such that $\alpha_{A}^{2}+\alpha_{B}^{2}=1$. For the 3-TS network coding, $G$ is given as follows.

$$
G=\sqrt{\frac{1}{\alpha_{B}^{2} P_{A}\left|h_{U}\right|^{2}+\alpha_{A}^{2} P_{B}\left|g_{U}\right|^{2}+\sigma^{2}}}
$$

The end-to-end SINRs for the 3-TS network coding are then calculated as follows.

$$
\begin{gathered}
\gamma_{A}=\frac{G^{2} P_{R}\left|h_{D}\right|^{2} \alpha_{A}^{2} P_{B}\left|g_{U}\right|^{2}}{G^{2} P_{R}\left|h_{D}\right|^{2} \psi_{3}+\left(\sum_{k} P_{I_{k}}^{(3)}\left|i_{A_{k}}^{(3)}\right|^{2}\right)+\sigma^{2}} \\
\gamma_{B}=\frac{G^{2} P_{R}\left|g_{D}\right|^{2} \alpha_{B}^{2} P_{A}\left|h_{U}\right|^{2}}{G^{2} P_{R}\left|g_{D}\right|^{2} \psi_{3}+\left(\sum_{k} P_{I_{k}}^{(3)}\left|i_{B_{k}}^{(3)}\right|^{2}\right)+\sigma^{2}} \\
\psi_{3}=\alpha_{B}^{2}\left(\sum_{k} P_{I_{k}}^{(1)}\left|i_{R_{k}}^{(1)}\right|^{2}+\sigma^{2}\right)+ \\
\alpha_{A}^{2}\left(\sum_{k} P_{I_{k}}^{(2)}\left|i_{R_{k}}^{(2)}\right|^{2}+\sigma^{2}\right)
\end{gathered}
$$

3) 3-TS Network Coding with Maximum Ratio Combining $(M R C)$ : As shown in Figure 2 and Figure 3, the 3-TS NWC scheme with MRC takes advantage of the direct links provided by unicasting D2D communications in a 3-TS network coding operation. In the first time slot, source A transmits to both the relay node and node $\mathrm{B}$. The transmission to source B is done through the D2D link. Similarly, in the second time slot, source B transmits to both the relay node and node A. In the third time slot, network coded data is transmitted to both receiving nodes by the relay node. As a result, each destination node receives the information twice: first through the D2D link and then from the relay, and can, therefore, take advantage of receiver diversity.

Assuming that nodes A and B support MRC, the received SINR at A and B in the case of 3-TS NWC with MRC can be approximated as:

$$
\begin{gathered}
\gamma_{A, 3-T S, M R C} \approx \gamma_{A, 3-T S}+\frac{P_{B}\left|g_{U, \text { direct }}\right|^{2}}{\left(\sum_{k} P_{I_{k}}^{(2)}\left|i_{A_{k}}^{(2)}\right|^{2}\right)+\sigma^{2}} \\
\gamma_{B, 3-T S, M R C} \approx \gamma_{B, 3-T S}+\frac{P_{A}\left|h_{U, \text { direct }}\right|^{2}}{\left(\sum_{k} P_{I_{k}}^{(1)}\left|i_{B_{k}}^{(1)}\right|^{2}\right)+\sigma^{2}}
\end{gathered}
$$

where $\gamma_{A, 3-T S}$ and $\gamma_{B, 3-T S}$ are the received SINR in the traditional 3-TS scheme as calculated in (11) and (12). Channels $h_{U \text {,direct }}$ and $g_{U, \text { direct }}$ are direct channels from A to $\mathrm{B}$ and $\mathrm{B}$ to $\mathrm{A}$ respectively. 
4) Four-Time Slot (4-TS) Network Coding: In the 4-TS network coding, there are two distinct relay gains $\left(G_{A}\right.$ and $G_{B}$ ) that are given as follows.

$$
\begin{aligned}
G_{A} & =\sqrt{\frac{1}{P_{B}\left|g_{U}\right|^{2}+\sigma^{2}}} \\
G_{B} & =\sqrt{\frac{1}{P_{A}\left|h_{U}\right|^{2}+\sigma^{2}}}
\end{aligned}
$$

The end-to-end SINR values at sources $\mathrm{A}$ and $\mathrm{B}$ are calculated accordingly:

$$
\begin{gathered}
\gamma_{A}=\frac{G_{A}^{2} P_{R}\left|h_{D}\right|^{2} P_{B}\left|g_{U}\right|^{2}}{G_{A}^{2} P_{R}\left|h_{D}\right|^{2} \psi_{4, A}+\sum_{k} P_{I_{k}}^{(4)}\left|i_{A_{k}}^{(4)}\right|^{2}+\sigma^{2}} \\
\gamma_{B}=\frac{G_{B}^{2} P_{R}\left|g_{D}\right|^{2} P_{A}\left|h_{U}\right|^{2}}{G_{B}^{2} P_{R}\left|g_{D}\right|^{2} \psi_{4, B}+\sum_{k} P_{I_{k}}^{(2)}\left|i_{B_{k}}^{(2)}\right|^{2}+\sigma^{2}} \\
\psi_{4, A}=\left(\sum_{k} P_{I_{k}}^{(3)}\left|i_{R_{k}}^{(3)}\right|^{2}+\sigma^{2}\right) \\
\psi_{4, B}=\left(\sum_{k} P_{I_{k}}^{(1)}\left|i_{R_{k}}^{(1)}\right|^{2}+\sigma^{2}\right)
\end{gathered}
$$

5) Unicasting D2D Communication: Unicasting D2D communications allow the transmitting nodes to be aware of there respective intended receivers, and thereby facilitate controlling the transmit power levels such that the SINR at the receiving nodes can be controlled. Due to the direct D2D link, this transmission scheme is a bidirectional direct transmission, without involving instructire nodes such as an eNB or relay node. It is assumed that a transmission in one direction requires one time slot, which results in two time slots needed in total. The transmission from source A to source B takes place in the first time slot. Then, the received signal at source $\mathrm{B}$ is given as follows.

$$
y_{B}=\underbrace{\sqrt{P_{A}} h_{u} x_{A}}_{\text {desired signal }}+\left(\sum_{k} \sqrt{P_{I_{k}}^{(1)}} i_{B_{k}}^{(1)} x_{i_{k}}^{(1)}\right)+n_{B}
$$

The transmission from source B to source A takes place in the second time slot. The received signal at source $\mathrm{A}$ is given as follows.

$$
y_{A}=\underbrace{\sqrt{P_{B}} g_{u} x_{B}}_{\text {desired signal }}+\left(\sum_{k} \sqrt{P_{I_{k}}^{(2)}} i_{A_{k}}^{(2)} x_{i_{k}}^{(2)}\right)+n_{A}
$$

Taking the ratio of the desired signal's power over interference and noise power, the SINR values at sources A and B can be calculated as follows.

$$
\begin{aligned}
\gamma_{A} & =\frac{P_{B}\left|g_{U}\right|^{2}}{\left(\sum_{k} P_{I_{k}}^{(2)}\left|i_{A_{k}}^{(2)}\right|^{2}\right)+\sigma^{2}} \\
\gamma_{B} & =\frac{P_{A}\left|h_{U}\right|^{2}}{\left(\sum_{k} P_{I_{k}}^{(1)}\left|i_{B_{k}}^{(1)}\right|^{2}\right)+\sigma^{2}}
\end{aligned}
$$

\section{Proposed Resource Allocation And Mode SELECTION Algorithms}

\section{A. Resource Allocation}

The resource allocation algorithm proposed in this paper takes into consideration the number of required resources by the different local communication schemes discussed above, and makes use of resource utilization counters. The basic idea of the proposed resource allocation and mode selection schemes is to allocate the resources which are least used, and thereby to avoid assigning a high number of communication links to the same resource. We assume that the eNB has knowledge of which UL and DL resources are used by all UEs within its coverage area. This assumption is realistic, in, for example, LTE networks, in which UEs regularly send measurement reports to their serving eNBs even when operating in D2D mode. Let us define UL and DL utilization vectors $\rho_{\mathbf{U}}$ and $\rho_{\mathbf{D}}$, and denote $\rho_{U}(i)$ as the utilization counter of UL resource $i$ and $\rho_{D}(j)$ as the utilization counter of DL resource $j$. For each communicating UE pair, UE-A and UE-B, the eNB needs to select two UL resources and two DL resources (denoted by $U 1$, $U 2, D 1$, and $D 2$ respectively), that are candidates of allocated resources for a two-way communication between UE-A and UE-B.

The eNB executes the following procedure. Note that this procedure is fundamentally similar to the scheduling procedure of an LTE eNB, but takes into account the specific characteristics of the transmission schemes discussed in this paper.

1) Selects the first UL resource, $U 1$, by choosing an UL resource $i$ randomly or taking into account the instantaneous channel response out of the resources for which $\rho_{U}(i)=\min \left(\rho_{\mathbf{U}}\right)$.

2) Increments $\rho_{U}(i), \rho_{U}(i) \leftarrow \rho_{U}(i)+1$.

3) Selects the second UL resource, $U 2$, by choosing an UL resource $i$ randomly out of the resources for which $\rho_{U}(i)=\min \left(\rho_{\mathbf{U}}\right)$.

4) Increments $\rho_{U}(i), \rho_{U}(i) \leftarrow \rho_{U}(i)+1$.

5) Selects the first DL resource, $D 1$, by choosing a DL resource $j$ randomly out of the resources for which $\rho_{D}(j)=\min \left(\rho_{\mathbf{D}}\right)$.

6) Increments $\rho_{D}(j), \rho_{D}(j) \leftarrow \rho_{D}(j)+1$.

7) Selects the second DL resource, $D 2$, by choosing a DL resource $j$ randomly out of the resources for which $\rho_{D}(j)=\min \left(\rho_{\mathbf{D}}\right)$.

8) Increments $\rho_{D}(j), \rho_{D}(j) \leftarrow \rho_{D}(j)+1$.

At the end of this resource balancing allocation procedure, four resources $(U 1, U 2, D 1$, and $D 2)$ are selected, and the resource utilization counters are updated. 


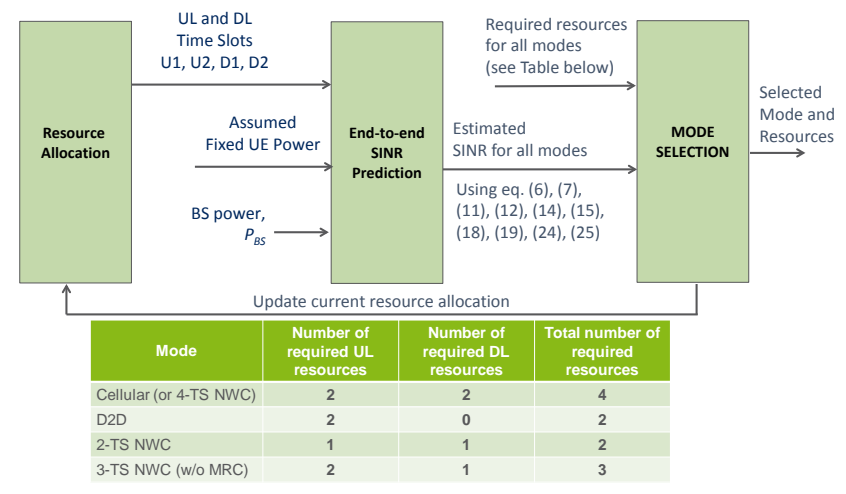

Fig. 6. The proposed mode selection algorithms and their interplay with resource allocation. The mode selection algorithms take the predicted end-toend SINR and the required resources per mode as its basic input and selects the mode that yields the highest expected SINR or highest spectral efficiency.

\section{B. Mode Selection (MS)}

Based on Figure 4 and the signal model, it is clear that there is an inherent trade-off between the number of used time slots, used total transmission power, and the resulting SINR levels and the achieved spectral and energy efficiency. This suggests that we should study two MS algorithms that aim at maximizing the achieved SINR (MS-NWC 1) and the spectral efficiency (MS-NWC 2), respectively.

In the proposed MS schemes, the eNB makes a prediction of the end-to-end SINR for all available transmission modes based on the channel knowledge, selected resources, and assuming appropriate eNB and UE transmit power levels (Figure 6). At this prediction stage, all UEs are assumed to transmit with a constant power of $P$, since it can be assumed that the resource allocation and mode selection take place before power control is performed. For the eNB to be able to make an end-to-end SINR prediction, it employs a mathematical model and computation technique that is characteristic to the specific transmission mode, as described in Section IV-B.

1) MS-NWC 1 (SINR-Maximizing Mode Selection): According to the SINR-maximizing MS scheme, the eNB selects the mode that has the highest predicted end-to-end SINR.

2) MS-NWC 2 (Spectral Efficiency-Maximizing Mode Selection): According to the spectral efficiency maximizing MS scheme, the eNB selects the mode that has the highest predicted spectral efficiency, $\hat{S}$. This prediction takes the endto-end SINR prediction $\left(\hat{\gamma}_{\text {mode }}\right)$ as well as the number of consumed resources $\left(\tau_{\text {mode }}\right)$ into account.

$$
\hat{S}_{\text {mode }}=\frac{\log _{2}\left(1+\hat{\gamma}_{\text {mode }}\right)}{\tau_{\text {mode }}}
$$

In (26), $\tau_{\text {mode }}$ is equal to 4 for cellular mode, 3 for classical physical layer NWC and NWC with MRC modes, and 2 for the 2-TS physical layer network coding and D2D modes.
TABLE I

SIMULATION PARAMETERS

\begin{tabular}{|l|c|}
\hline System bandwidth & $5 \mathrm{MHz}$ \\
\hline Carrier frequency & $2 \mathrm{GHz}$ \\
\hline Gain at 1 m distance & $-37 \mathrm{~dB}$ \\
\hline Path loss coefficient & 3.5 \\
\hline Log normal shadow fading $\sigma$ & $6 \mathrm{~dB}$ \\
\hline Number of Monte Carlo iterations & 100 \\
\hline Number of cells & 7 \\
\hline Number of UE pairs per cell & $500 \mathrm{~m}$ \\
\hline Number of radio resources per cell & $\sqrt{0.5}$ (both) \\
\hline Cell radius & $40 \mathrm{dBm}$ \\
\hline Power allocation numbers $\left(\alpha_{A}, \alpha_{B}\right)$ & 0.8 \\
\hline eNB transmit power & $-10 \mathrm{dBm}(\mathrm{MS}-\mathrm{NWC}$ 1), \\
\hline Path-loss compensation $\left(\alpha_{F P C}\right)$ & $20 \mathrm{dBm}(\mathrm{MS}-\mathrm{NWC}$ ) \\
\hline $\begin{array}{l}\text { Assumed constant UE power } \\
\text { for mode selection }(P)\end{array}$ & \\
\hline
\end{tabular}

\section{NUMERICAL RESULTS}

\section{A. Simulation Setup and Operation Modes}

Mode selection in a multi-cell environment is a complex problem that can be advantageously studied by system level simulations $[18,22]$. To obtain numerical results, we use a realistic system level simulator called the Rudimentary Network (RUNE) simulator [30] that was enhanced to support NWC, unicasting D2D communications and the proposed mode selection schemes. We consider a 7-cell network and collect statistics using Monte Carlo simulations. In each Monte Carlo experiment, a cellular system is generated with 7 hexagonal cells, a fixed number of locally communicating UE pairs per cell, and a fixed number of radio resources (resource blocks) per cell. The UEs are dropped randomly within the cell with uniform distribution. Additionally, we consider both low traffic and high traffic scenarios. The simulation parameters are listed in Table I.

We assume that the system employs LTE open-loop fractional path loss compensation power control with path-loss compensation factor $\alpha_{F P C}$ given in Table I. Power control is assumed to be done taking into account the selected resources, as proposed in [8].

To compare the performance of the communication schemes discussed in this paper, seven operating modes are considered in our simulations.

1) 2-TS NWC: All UE pairs use the 2-TS network coding scheme.

2) 3-TS NWC: All UE pairs use the 3-TS network coding scheme with maximum ratio combining.

3) 4-TS NWC: All UE pairs use the 4-TS network coding scheme (traditional cellular mode).

4) D2D - No NWC: All UE pairs are forced to communicate in unicasting D2D mode.

5) $M S-N W C 1$ : End-to-end SINR-maximizing mode selection where it is possible to choose any of the transmission schemes in Figure 4.

6) $M S-N W C 2$ : Spectral efficiency-maximizing mode selection where it is possible to choose any of the transmission schemes in Figure 4. 


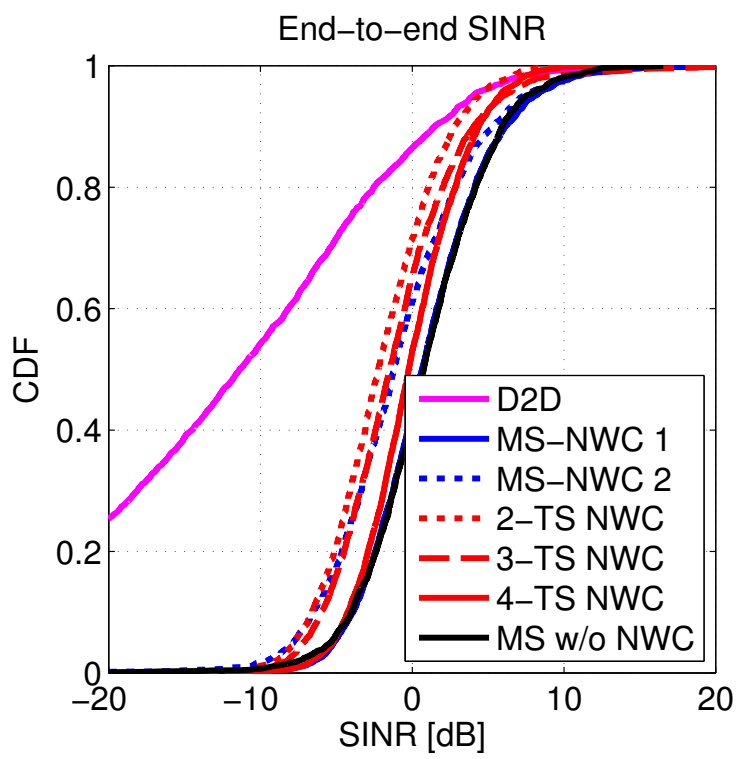

Fig. 7. End-to-end SINR performance of the available local transmission schemes, low traffic scenario (4 UE pairs per cell).

7) MS without NWC: A mode selection strategy where it is possible to choose either D2D or cellular (4-TS mode). Network coding is not supported. The selection is based on a simple channel gain comparison, i.e. if the channel between the transmitting UE and the receiving UE has higher gain than the channel between the transmitting $\mathrm{UE}$ and the eNB, D2D mode is selected. Otherwise, cellular mode is selected.

\section{B. Numerical Results and Discussion}

1) Behaviour of the Mode Selection Algorithm: Figure 13 and Table II show the transmission modes selected by the mode selection algorithm in two different traffic scenarios. We can observe that the SINR-maximizing mode selection tends to choose a transmission scheme that consumes more resources in an attempt to reduce interference, while the spectral-efficiency maximizing mode selection chooses recourse-efficient modes (2-TS NWC or D2D mode) most of the time.

2) End-to-End SINR: Figure 7 compares the SINR performance of the transmission schemes of Figure 2, when using the proposed MS algorithms that support both NWC and D2D (MS-NWC 1 and MS-NWC 2) and MS in an integrated D2Dcellular network (MS without NWC). As expected, the SINR is maximized when using MS-NWC 1 . On the other hand, the gain of employing NWC in an integrated cellular and D2D network in terms of SINR is negligible.

However, as shown in 8, MS-NWC 1 performs poorly when the interference level is high. This is caused by inaccurate SINR prediction resulted from assuming constant power for all UEs. In high traffic situation, it is better to employ MS-NWC 2 because the inaccuracy of SINR prediction is suppressed by logarithmic function in the mode selection criterion. In any case, Figure 7 and Figure 8 prove that an integrated D2D-

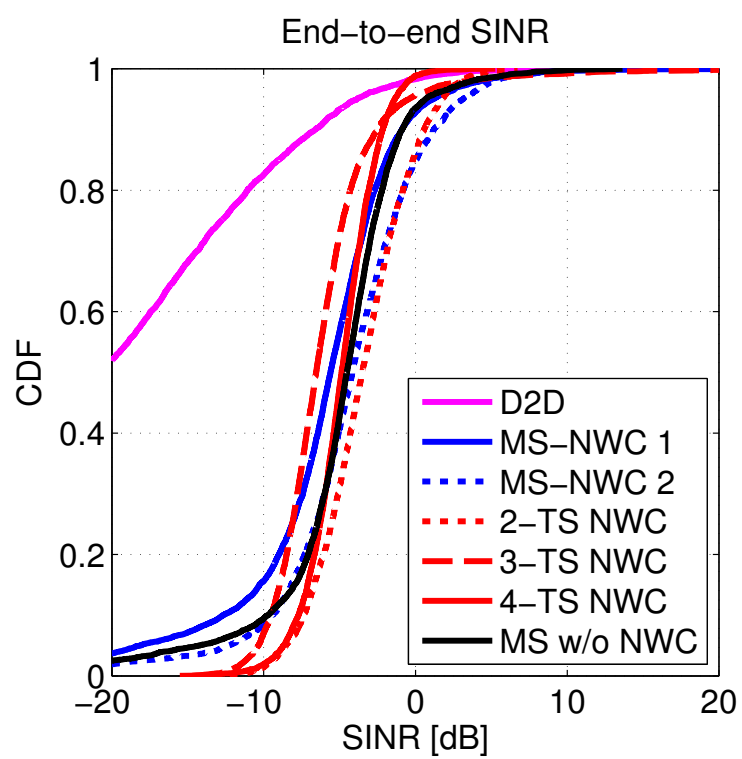

Fig. 8. End-to-end SINR performance of the transmission schemes under study, high traffic scenario (8 UE pairs per cell).

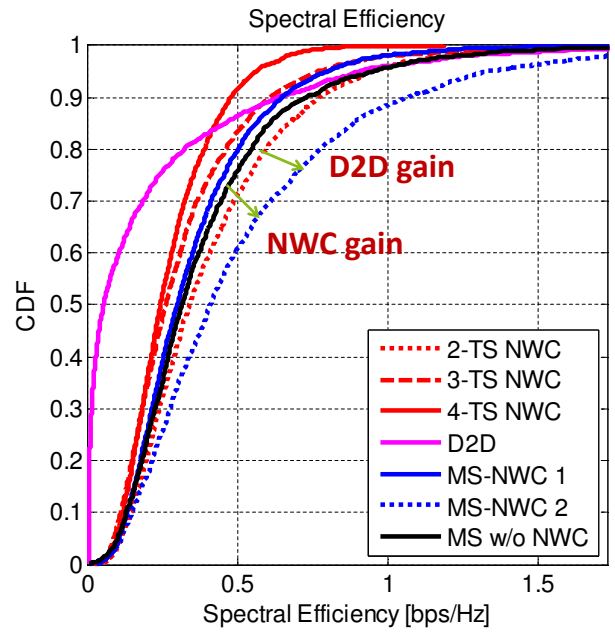

Fig. 9. Spectral efficiency of the transmission schemes under study, low traffic scenario (4 UE pairs per cell).

NWC-cellular network can always achieve high end-to-end SINR with a proper mode selection.

3) Spectrum Efficiency: As Figure 9 shows, however, NWC leads to significant spectral efficiency increase if the mode selection scheme of MS-NWC 2 is employed. This clearly suggests that the main benefit of introducing NWC into an integrated D2D-cellular network is further improving the spectral efficiency. MS-NWC 2 also outperforms other mode selection strategies in the high traffic scenario as shown in Figure 10.

4) Invested Transmit Power: Finally, as suggested by Figure 11 and Figure 12, this high spectral efficiency can be obtained even at low power consumption when using proper 


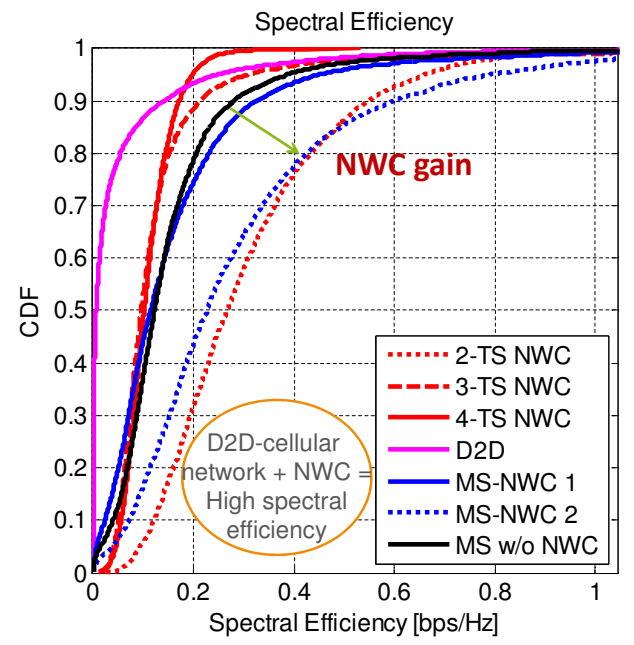

Fig. 10. Spectral efficiency of the transmission schemes under study, high traffic scenario (8 UE pairs per cell).

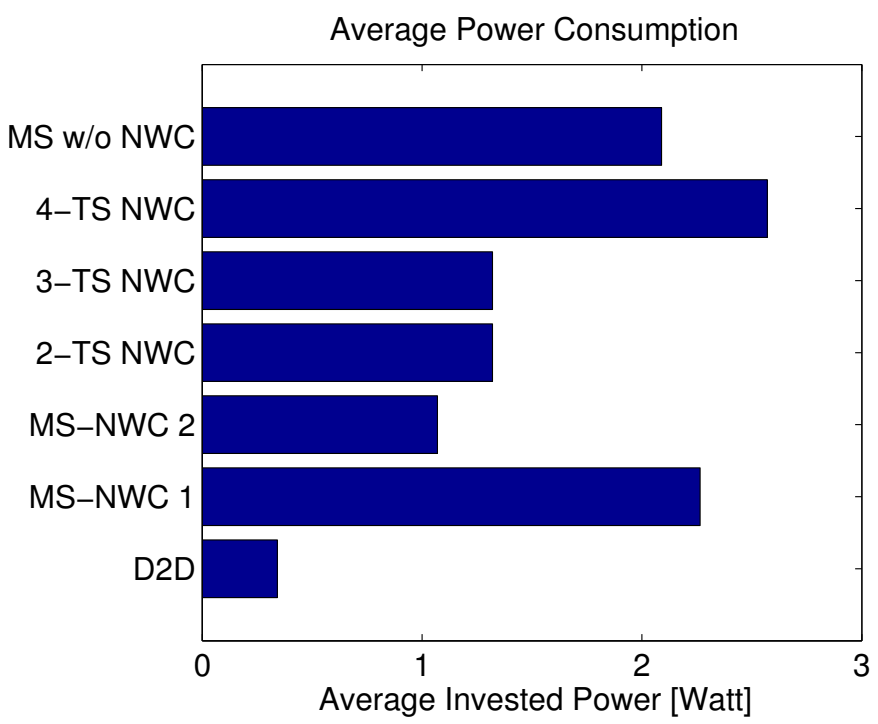

Fig. 11. Average power consumption of the transmission schemes under study, low traffic scenario (4 UE pairs per cell).

mode selection (see MS-NWC 2 column). These results indicate that a network that supports both D2D and NWC is more energy-efficient than a network that supports NWC only. In other words, the expected gain that $\mathrm{D} 2 \mathrm{D}$ brings in a cellular network employing NWC is energy efficiency.

\section{CONCLUSIONS}

In this paper, we asked whether unicasting D2D and NWC schemes can or should be used together in scenarios in which local (proximate) communication opportunities exist. This question is particularly opportune when standardization bodies, such as the 3GPP are considering introducing unicasting D2D communications support in the upcoming releases of LTE networks. We have shown that introducing NWC in integrated D2D-cellular networks can provide significant spectral

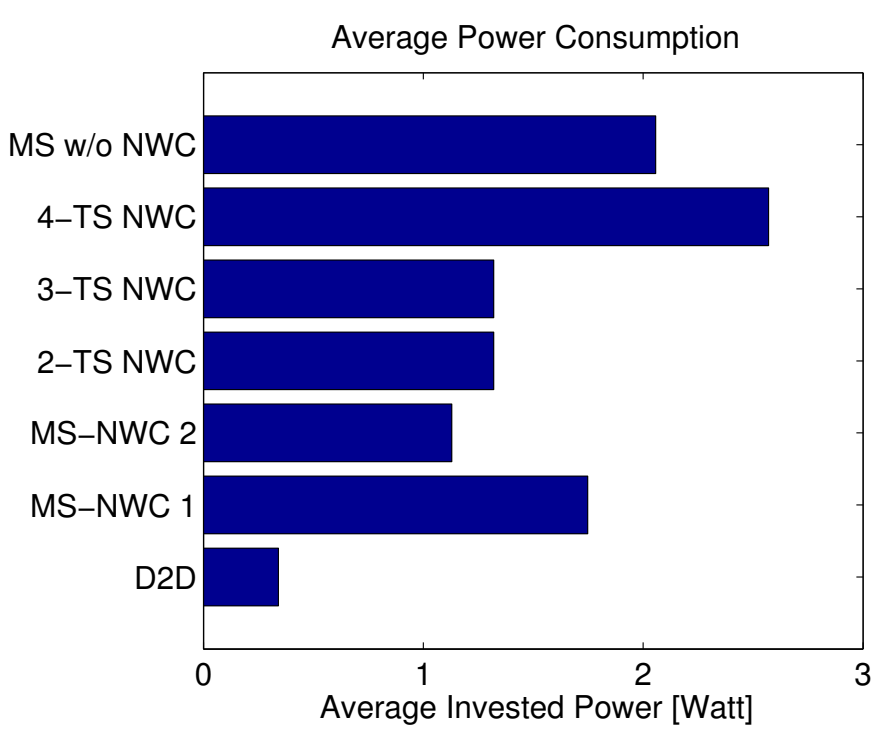

Fig. 12. Average power consumption of the transmission schemes under study, high traffic scenario (8 UE pairs per cell).

Low Load:
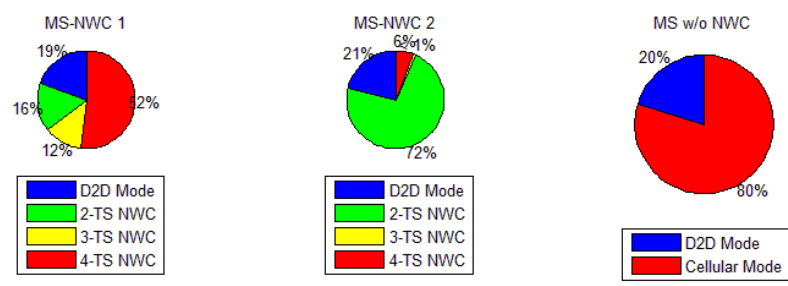

High Load:
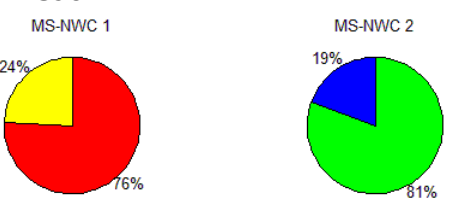

MS w/o NWC
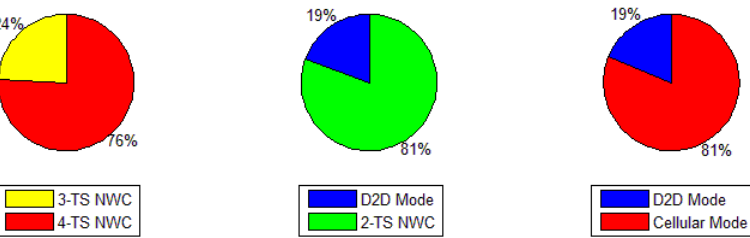

Fig. 13. Mode selection statistics at low and high network load.

TABLE II

MODE SELECTION OUTPUTS IN LOW AND HIGH TRAFFIC SCENARIOS

\begin{tabular}{|c|c|c|}
\hline Mode selection & Low traffic & High traffic \\
\hline $\begin{array}{l}1 \\
\text { ximizing) }\end{array}$ & $\begin{array}{l}\text { 3-TS NWC: } 24 \% \\
\text { 4-TS NWC: } 76 \%\end{array}$ & $\begin{array}{l}\text { D2D mode: } 19 \% \\
\text { 2-TS NWC: } 16 \% \\
\text { 3-TS NWC: } 12 \% \\
\text { 4-TS NWC: } 52 \%\end{array}$ \\
\hline $\begin{array}{l}\text { 2 } \\
\text { m efficiency } \\
\text { ing) }\end{array}$ & $\begin{array}{l}\text { D2D mode: } 19 \% \\
\text { 2-TS NWC: } 81 \%\end{array}$ & $\begin{array}{l}\text { D2D mode: } 21 \% \\
\text { 2-TS NWC: } 72 \% \\
\text { 3-TS NWC: } 1 \% \\
\text { 4-TS NWC: } 6 \%\end{array}$ \\
\hline$\overline{M S} \mathbf{v}$ & $\begin{array}{c}\text { D2D mode: } 19 \% \\
\text { Cellular: } 81 \%\end{array}$ & $\begin{array}{l}\text { D2D mode: } 20 \% \\
\text { Cellular: } 80 \%\end{array}$ \\
\hline
\end{tabular}


efficiency gain. On the other hand, introducing unicasting D2D in a cellular network that employs NWC reduces the invested transmit power. Our results show that D2D and NWC can complement each other and be advantageously used jointly, provided that the network applies a proper mode selection algorithm. Our planned future work includes aspects that are beyond the scope of this paper, including the modeling of the impact of channel state information imperfections, as well as the modeling of other imperfections, such as synchronization errors or scheduling delays.

\section{ACKNOWLEDGMENTS}

I am grateful to Aidilla Pradini, at Ericsson AB, for the many fruitful discussions, continuous encouragement and helping with the simulations throughout this study.

\section{APPENDIX: DETAILED SINR ANALYSIS}

\section{A. Two-Time Slot (2-TS) Network Coding}

The signal received at the relay in the first time slot is expressed as follows.

$r=\sqrt{P_{A}} h_{U} x_{A}+\sqrt{P_{B}} g_{U} x_{B}+\left(\sum_{k} \sqrt{P_{I_{k}}^{(1)}} i_{R_{k}}^{(1)} x_{i_{k}}^{(1)}\right)+n_{R}$

After receiving signals from both sources, the relay transmits its network coded signals to sources A and B. The signal received at each source in the second time slot is expressed as follows:

$$
\begin{aligned}
& y_{A}=G \sqrt{P_{R}} h_{D} r+\left(\sum_{k} \sqrt{P_{I_{k}}^{(2)}} i_{A_{k}}^{(2)} x_{i_{k}}^{(2)}\right)+n_{A} \\
& y_{B}=G \sqrt{P_{R}} g_{D} r+\left(\sum_{k} \sqrt{P_{I_{k}}^{(2)}} i_{B_{k}}^{(2)} x_{i_{k}}^{(2)}\right)+n_{B}
\end{aligned}
$$

We assume that $h_{U}$ and $h_{D}$ are known to source A, $g_{U}$ and $g_{D}$ are known to source B, while $G$ and $P_{R}$ are known to both sources and each source also knows its own data symbols. As a consequence, the interference terms whose components are known to the receiver can be excluded in the SINR calculation. Substituting (27) into (28) and (29) gives:

$$
\begin{aligned}
y_{A}= & \underbrace{G \sqrt{P_{R}} h_{D} \sqrt{P_{A}} h_{U} x_{A}}_{\text {known by source } \mathrm{A}}+\underbrace{G \sqrt{P_{R}} h_{D} \sqrt{P_{B}} g_{U} x_{B}}_{\text {desired signal }} \\
& +G \sqrt{P_{R}} h_{D}\left(\sum_{k} \sqrt{P_{I_{k}}^{(1)}} i_{R_{k}}^{(1)} x_{i_{k}}^{(1)}\right)+ \\
& G \sqrt{P_{R}} h_{D} n_{R}+\left(\sum_{k} \sqrt{P_{I_{k}}^{(2)}} i_{A_{k}}^{(2)} x_{i_{k}}^{(2)}\right)+n_{A}
\end{aligned}
$$

$$
\begin{aligned}
y_{B}= & \underbrace{G \sqrt{P_{R}} g_{D} \sqrt{P_{A}} h_{U} x_{A}}_{\text {desired signal }}+\underbrace{G \sqrt{P_{R}} g_{D} \sqrt{P_{B}} g_{U} x_{B}}_{\text {known by source B }} \\
& +G \sqrt{P_{R}} g_{D}\left(\sum_{k} \sqrt{P_{I_{k}}^{(1)}} i_{R_{k}}^{(1)} x_{i_{k}}^{(1)}\right)+ \\
& G \sqrt{P_{R}} g_{D} n_{R}+\left(\sum_{k} \sqrt{P_{I_{k}}^{(2)}} i_{B_{k}}^{(2)} x_{i_{k}}^{(2)}\right)+n_{B}
\end{aligned}
$$

Taking the ratio of desired signal's power over interference and noise power, while excluding the known signals from the interference, end-to-end SINR values at sources A and B are written according to (6), (7), and (8).

\section{B. Three-Time Slot (3-TS) Network Coding}

In the first time slot, source A transmits its signal to the relay. Signal received at the relay in the first time slot can be expressed as follows.

$$
r_{A}=\sqrt{P_{A}} h_{U} x_{A}+\left(\sum_{k} \sqrt{P_{I_{k}}^{(1)}} i_{R_{k}}^{(1)} x_{i_{k}}^{(1)}\right)+n_{R}^{(1)}
$$

In the second time slot, source B transmits its signal to the relay. Signal received at the relay in the second time slot:

$$
r_{B}=\sqrt{P_{B}} g_{U} x_{B}+\left(\sum_{k} \sqrt{P_{I_{k}}^{(2)}} i_{R_{k}}^{(2)} x_{i_{k}}^{(2)}\right)+n_{R}^{(2)}
$$

In the third time slot, the relay simultaneously transmit network coded signals to sources A and B. Signals received at sources A and B in the third time slot is expressed as follows.

$$
\begin{aligned}
y_{A}= & G \sqrt{P_{R}} h_{D}\left(\alpha_{B} r_{A}+\alpha_{A} r_{B}\right)+ \\
& \left(\sum_{k} \sqrt{P_{I_{k}}^{(3)}} i_{A_{k}}^{(3)} x_{i_{k}}^{(3)}\right)+n_{A} \\
y_{B}= & G \sqrt{P_{R}} g_{D}\left(\alpha_{B} r_{A}+\alpha_{A} r_{B}\right)+ \\
& \left(\sum_{k} \sqrt{P_{I_{k}}^{(3)}} i_{B_{k}}^{(3)} x_{i_{k}}^{(3)}\right)+n_{B}
\end{aligned}
$$

Substitution of (32) and (33) into (34) and (35) yields the following expressions.

$$
\begin{aligned}
y_{A}= & \underbrace{G \sqrt{P_{R}} h_{D} \alpha_{B} \sqrt{P_{A}} h_{U} x_{A}}_{\text {known by source A }}+ \\
& G \sqrt{P_{R}} h_{D} \alpha_{B}\left(\sum_{k} \sqrt{P_{I_{k}}^{(1)}} i_{R_{k}}^{(1)} x_{i_{k}}^{(1)}\right)+ \\
& G \sqrt{P_{R}} h_{D} \alpha_{B} n_{R}^{(1)}+\underbrace{G \sqrt{P_{R}} h_{D} \alpha_{A} \sqrt{P_{B}} g_{U} x_{B}}_{\text {desired signal }}+ \\
& G \sqrt{P_{R}} h_{D} \alpha_{A}\left(\sum_{k} \sqrt{P_{I_{k}}^{(2)}} i_{R_{k}}^{(2)} x_{i_{k}}^{(2)}\right)+ \\
& G \sqrt{P_{R}} h_{D} \alpha_{A} n_{R}^{(2)}+ \\
& \left(\sum_{k} \sqrt{P_{I_{k}}^{(3)}} i_{A_{k}}^{(3)} x_{i_{k}}^{(3)}\right)+n_{A}
\end{aligned}
$$




$$
\begin{aligned}
y_{B}= & \underbrace{G \sqrt{P_{R}} g_{D} \alpha_{B} \sqrt{P_{A}} h_{U} x_{A}}_{\text {desired signal }}+ \\
& G \sqrt{P_{R}} g_{D} \alpha_{B}\left(\sum_{k} \sqrt{P_{I_{k}}^{(1)}} i_{R_{k}}^{(1)} x_{i_{k}}^{(1)}\right)+ \\
& G \sqrt{P_{R}} g_{D} \alpha_{B} n_{R}^{(1)}+\underbrace{G \sqrt{P_{R}} g_{D} \alpha_{A} \sqrt{P_{B}} g_{U} x_{B}}_{\text {known by source B }}+ \\
& G \sqrt{P_{R}} g_{D} \alpha_{A}\left(\sum_{k} \sqrt{P_{I_{k}}^{(2)}} i_{R_{k}}^{(2)} x_{i_{k}}^{(2)}\right)+ \\
& G \sqrt{P_{R}} g_{D} \alpha_{A} n_{R}^{(2)}+ \\
& \left(\sum_{k} \sqrt{P_{I_{k}}^{(3)}} i_{B_{k}}^{(3)} x_{i_{k}}^{(3)}\right)+n_{B}
\end{aligned}
$$

Taking the ratio of desired signal's power over interference and noise power, while excluding the known signals from the interference, end-to-end SINR values at sources A and B are calculated as written in (11), (12), and (13).

\section{Four-Time Slot (4-TS) Network Coding}

The first and second time slots are allocated for communication from source A to source B. In the first time slot, source A transmits $x_{A}$ to the relay, and the relay received $r_{A}$.

$$
r_{A}=\sqrt{P_{A}} h_{U} x_{A}+\left(\sum_{k} \sqrt{P_{I_{k}}^{(1)}} i_{R_{k}}^{(1)} x_{i_{k}}^{(1)}\right)+n_{R}^{(1)}
$$

In the second time slot, the relay amplifies $r_{A}$ by gain $G_{B}$ and transmits to source B. The received signal at source B is expressed as follows.

$$
y_{B}=G_{B} \sqrt{P_{R}} g_{D} r_{A}+\left(\sum_{k} \sqrt{P_{I_{k}}^{(2)}} i_{B_{k}}^{(2)} x_{i_{k}}^{(2)}\right)+n_{B}
$$

The third and fourth time slots are allocated for communication from source B to source A. In the third time slot, source B transmits $x_{B}$ to the relay, and the relay received $r_{B}$.

$$
r_{B}=\sqrt{P_{B}} g_{U} x_{B}+\left(\sum_{k} \sqrt{P_{I_{k}}^{(3)}} i_{R_{k}}^{(3)} x_{i_{k}}^{(3)}\right)+n_{R}^{(3)}
$$

In the fourth time slot, the relay amplifies $r_{B}$ by gain $G_{A}$ and transmits to source $\mathrm{A}$. The received signal at source $\mathrm{A}$ is expressed as follows.

$$
y_{A}=G_{A} \sqrt{P_{R}} h_{D} r_{B}+\left(\sum_{k} \sqrt{P_{I_{k}}^{(4)}} i_{A_{k}}^{(4)} x_{i_{k}}^{(4)}\right)+n_{A}
$$

Substitutions of (38) into (39) and (40) into (41) result in the following expressions.

$$
\begin{aligned}
y_{A}= & \underbrace{G_{A} \sqrt{P_{R}} h_{D} \sqrt{P_{B}} g_{U} x_{B}}_{\text {desired signal }}+ \\
& G_{A} \sqrt{P_{R}} h_{D}\left(\sum_{k} \sqrt{P_{I_{k}}^{(3)}} i_{R_{k}}^{(3)} x_{i_{k}}^{(3)}\right)+ \\
& G_{A} \sqrt{P_{R}} h_{D} n_{R}^{(3)}+\left(\sum_{k} \sqrt{P_{I_{k}}^{(4)}} i_{A_{k}}^{(4)} x_{i_{k}}^{(4)}\right)+n_{A} \\
y_{B}= & \underbrace{G_{B} \sqrt{P_{R}} g_{D} \sqrt{P_{A}} h_{U} x_{A}}_{\text {desired signal }}+ \\
& G_{B} \sqrt{P_{R}} g_{D}\left(\sum_{k} \sqrt{P_{I_{k}}^{(1)}} i_{R_{k}}^{(1)} x_{i_{k}}^{(1)}\right)+ \\
& G_{B} \sqrt{P_{R}} g_{D} n_{R}^{(1)}+\left(\sum_{k} \sqrt{P_{I_{k}}^{(2)}} i_{B_{k}}^{(2)} x_{i_{k}}^{(2)}\right)+n_{B}
\end{aligned}
$$

Taking the ratio of desired signal's power over interference and noise power, end-to-end SINRs are calculated as specified in (18), (19), (20), and (21).

\section{REFERENCES}

[1] G. Fodor, A. Pradini, and A. Gattami, "On applying network coding in network assisted device-to-device communications," in European Wireless, Barcelona, Spain, May 2014.

[2] K. Doppler, M. Rinne, C. Wijting, C. Ribeiro, and K. Hugl, "Device-to-device communication as an underlay to LTE-advanced networks," Communications Magazine, IEEE, vol. 47, no. 12, pp. 42 -49, dec. 2009.

[3] G. Fodor, E. Dahlman, G. Mildh, S. Parkvall, N. Reider, G. Miklós, and Z. Turányi, "Design aspects of network assisted device-to-device communications," IEEE Communications Magazine, vol. 50, no. 3, pp. 170 -177, March 2012.

[4] Y. Li, T. Wu, P. Hui, D. Jin, and S. Chen, "Social-aware d2d communications: Qualitative insights and quantitative analysis," IEEE Communications Magazine, pp. 150-158, June 2014.

[5] L. Lei, Z. Zhong, C. Lin, and X. Shen, "Operator controlled device-to-device communications in LTEadvanced networks," Wireless Communications, IEEE, vol. 19, no. 3, pp. $96-104$, june 2012.

[6] N. Lee, X. Lin, J. G. Andrews, and R. W. H. Jr., "Power control for D2D underlaid cellular networks: Modeling, algorithms and analysis," submitted to IEEE JSAC. arXiv:1305.6161 [cs.IT].

[7] G. Fodor and N. Reider, "A distributed power control scheme for cellular network assisted D2D communications," in Global Telecommunications Conference (GLOBECOM 2011), 2011 IEEE, dec. 2011, pp. 1 -6.

[8] G. Fodor, D. D. Penda, M. Belleschi, M. Johansson, and A. Abrardo, "A comparative study of power control approaches for device-to-device communications," in IEEE International Conference on Communications, ICC'13, 2013. 
[9] M. Belleschi, G. Fodor, and A. Abrardo, "Performance analysis of a distributed resource allocation sceheme for D2D communications," in IEEE Workshop on Machineto-Machine Communications. IEEE, 2011.

[10] A. Asadi, Q. Wang, and V. Mancuso, "A survey on device-to-device communication in cellular networks," IEEE Surveys on Communications, 2013, submitted to the IEEE Surveys on Communications, October 2013.

[11] 3GPP, "Scenarios and requirements for general use cases and national security and public safety," May 2013, Tech. Report 22.803.

[12] G. Fodor, S. Roger, N. Rajatheva, S. B. Slimane, T. Svensson, P. Popovski, J. M. B. da Silva Jr., and S. Ali, "An overview of device-to-device communications technology components in metis," IEEE Access, vol. 4, pp. 3288 - 3299, June 2016.

[13] R. H. Y. Louie, Y. Li, and B. Vucetic, "Practical physical layer network coding for two-way relay channels: performance analysis and comparison," Wireless Communications, IEEE Transactions on, vol. 9, no. 2, pp. 764-777, 2010.

[14] F. Yang, M. Huang, S. Zhang, and W. Zhou, "Performance analysis on two-way relay system with co-channel interference," Wireless Personal Communications, vol. 72, no. 1, pp. 415-434, 2013. [Online]. Available: http://dx.doi.org/10.1007/s11277013-1021-3

[15] G. Fodor, A. Pradini, and A. Gattami, "Device-to-device communications and network coding: Friends or foes?" IEEE Comsoc MMTC E-Letter, vol. 9, no. 1, pp. 33-35, January 2014.

[16] F. S. Tabataba, P. Sadeghi, C. Hucher, and M. R. Pakravan, "Impact of channel estimation errors and power allocation onanlog network coding and routing in two-way relaying," IEEE Transactions on Vehicular Technology, vol. 61, no. 7, pp. 3223-3238, September 2012.

[17] S. Yadav and P. K. Upadhyay, "Impact of outdated channel estimation on opportunistic two-way anc-based relaying with three-phase transmissions," IEEE Transactions on Vehicular Technology, vol. 64, no. 12, pp. 57505766, December 2015.

[18] K. Doppler, C. Yu, C. Ribeiro, and P. Janis, "Mode selection for device-to-device communication underlaying an LTE-advanced network," in IEEE Wireless Communications and Networking Conference (WCNC), 2010, pp. $1-6$.

[19] K. Akkarajitsakul, P. Phunchongharn, E. Hossain, and V. K. Bhargava, "Mode selection for energy-efficient D2D communications in lte-advanced networks: A conditional game approach," in IEEE International Conference on Communication Systems, Nov 2012, pp. 488-492.

[20] S. Maghsudi and S. Stanczak, "Transmission mode selection for network-assisted device to device communication: A levy-bandit approach," in IEEE International Conference on Acoustic, Speech and Signal Processing (ICASSP), 2014, pp. 7009-7013.
[21] A. Morattab, Z. Dziong, K. Sohraby, and M. H. Islam, "An optimal mimo mode selection method for $\mathrm{d} 2 \mathrm{~d}$ transmission in cellular networks," in IEEE 11th International Conference on Wireless and Mobile Computing, Networking and Communications (WiMob), 2015, pp. 392398.

[22] Y. Liu, "Optimal mode selection in D2D-enabled multibase station systems," IEEE Communications Letters, vol. 20, no. 3, pp. 470-473, March 2016.

[23] S. Maghsudi and D. Niyato, "On transmission mode selection in D2D-enhanced small cell networks," IEEE Wireless Communications Letters, vol. 6, no. 5, pp. 618621, Oct 2017.

[24] L. Lei and Y. Kuang, Smart Device to Smart Device Communication, S. Mumtaz and J. Rodriguez, Eds. Springer International Publishing, 2014, iSBN 978-3-319-049625.

[25] M. Rodziewicz, "Network coding aided device-to-device communication," in European Wireless, 2012. EW. 18th European Wireless Conference, 2012, pp. 1-5.

[26] Z. Zhao, S. Shao, and J. Sun, "The research of network coding in $\mathrm{d} 2 \mathrm{~d}$ communication system," in International Conference on Computer, Networks, and Communication Engineering (ICCNCE'13). Atlantis Press, 2013.

[27] Y. Zhao, Y. Li, and N. Ge, "Physical layer network coding aided two-way device-to-device communication underlaying cellular networks," in IEEE Globecom, San Diego, USA., 6-10 December 2015.

[28] Y. Zhao, Y. Li, Z. Ding, N. Ge, and H. V. Poor, "A coalitional graph game framework for network coding-aided d2d communication," EURASIP Journal on Advances in Signal Processing, vol. 2, 2016.

[29] P. Pahlevani, M. Hundeboll, M. V. Pedersen, D. E. Lucani, H. Charaf, F. H. P. Fitzek, H. Bagheri, and M. D. Katz, "Novel concepts for device-to-device communication using network coding," IEEE Communications Magazine, vol. 52, no. 4, pp. 32-39, April 2014.

[30] J. Zander and S. L. Kim, Radio Resource Management for Wireless Networks, J. Zander, Ed. Artech House, 2001. 